Groups Geom. Dyn. 6 (2012), 389-407

DOI $10.4171 / \mathrm{GGD} / 161$
Groups, Geometry, and Dynamics

(C) European Mathematical Society

\title{
Universal Borel actions of countable groups
}

\author{
Simon Thomas*
}

\begin{abstract}
If the countable group $G$ has a nonabelian free subgroup, then there exists a standard Borel $G$-space such that the corresponding orbit equivalence relation is countable universal. In this paper, we will consider the question of whether the converse also holds.
\end{abstract}

Mathematics Subject Classification (2010). 03E15, 37A20.

Keywords. Borel equivalence relation, superrigidity, sofic groups.

\section{Introduction}

A Borel equivalence relation $E$ on a standard Borel space $X$ is said to be countable if every $E$-class is countable. For example, if $G$ is a countable group and $X$ is a standard Borel $G$-space, then the corresponding $G$-orbit equivalence relation $E_{G}^{X}$ is a countable Borel equivalence relation. Conversely, by a remarkable result of Feldman-Moore [6], if $E$ is an arbitrary countable Borel equivalence relation on the standard Borel space $X$, then there exists a countable group $G$ and a Borel action of $G$ on $X$ such that $E=E_{G}^{X}$.

Definition 1.1. A countable group $G$ is said to be action universal if there exists a standard Borel $G$-space $X$ such that $E_{G}^{X}$ is universal.

Recall that a countable Borel equivalence relation $E$ is said to be universal if $F \leq_{B} E$ for every countable Borel equivalence relation $F$. (In this case, we will often say that $E$ is countable universal.) For example, by Dougherty-JacksonKechris [4], if the countable group $G$ has a nonabelian free subgroup, then $G$ is action universal. More precisely, for each countable group $G$ and standard Borel space $X$, let $E(G, X)$ be the orbit equivalence relation arising from the shift action of $G$ on the standard Borel space $X^{G}$. Note that this notation includes the cases when $X$ is a finite or a countably infinite standard Borel space. For example, $E(G, 2)$ is the orbit equivalence relation arising from the shift action of $G$ on $2^{G}$.

\footnotetext{
* Research partially supported by NSF Grant DMS 0600940.
} 
Theorem 1.2 (Dougherty-Jackson-Kechris [4]). If the countable group $G$ has a nonabelian free subgroup, then $E(G, 2)$ is universal and hence $G$ is action universal.

No other examples of action universal groups are currently known. On the other hand, the following result is an immediate consequence of the results in JacksonKechris-Louveau [10, Section 2].

Theorem 1.3 (Jackson-Kechris-Louveau [10]). If $G$ is a countable amenable group, then $G$ is not action universal.

This raises the possibility of yet another "dynamic" version of the so-called von Neumann Conjecture that a countable group $G$ is non-amenable if and only if $G$ contains a copy of the free group $\mathbb{F}_{2}$ on two generators. (The original von Neumann conjecture, which is actually due to Day, was disproved by Ol'shanskii [16] in 1980. For other possible "dynamic" versions, see Jackson-Kechris-Louveau [10, 6.1(D)] and Kechris-Miller [12, Problem 28.14]. We should also mention the remarkable recent result of Gaboriau-Lyons [7] which states that if $G$ is a countable non-amenable group, then there is a free standard Borel $G$-space $X$ with a $G$-invariant ergodic probability measure $\mu$ such that there exists a free ergodic Borel action of $\mathbb{F}_{2}$ on $(X, \mu)$ with $E_{\mathbb{F}_{2}}^{X} \subseteq E_{G}^{X}$.)

Question 1.4. Is it true that if $G$ is a countable group, then the following statements are equivalent:

(i) $G$ is action universal.

(ii) $G$ contains a nonabelian free subgroup.

A positive answer to Question 1.4 seems extremely unlikely; and in Section 5, we will present some evidence which suggests that free Burnside groups of sufficiently large odd exponent are counterexamples.

Conjecture 1.5. If $n$ is a sufficiently large odd integer, then the free 2-generator Burnside group $B(2, n)$ of exponent $n$ is action universal.

Of course, it is also natural to ask whether every countable non-amenable group is action universal. However, in Section 4, we will prove that this is not true. (As we will explain in Section 4, Theorem 1.6 is a consequence of Theorem 4.7.)

Theorem 1.6. There exists a countable non-amenable group which is not action universal.

In the final section of this paper, we will switch our attention from universal actions to $G$-universal actions. Here if $G$ is a countable group and $X$ is a standard Borel $G$-space, then $E_{G}^{X}$ is said to be $G$-universal if $E_{G}^{Z} \leq_{B} E_{G}^{X}$ for every standard Borel 
$G$-space $Z$. In [4], Dougherty-Jackson-Kechris proved that if $G$ is any countable group, then $E\left(G, 2^{\mathbb{N}}\right)$ is $G$-universal; and in [10], Jackson-Kechris-Louveau proved that if $G$ is any countable group, then $E(G, \mathbb{N})$ is $G$-universal. However, there are currently no countable groups $G$ for which it is known that $E(G, 2)$ is not $G$-universal.

By Theorem 1.2, if $G$ has a nonabelian free subgroup, then $E(G, 2)$ is universal and hence $E(G, 2)$ is $G$-universal. On the other hand, suppose that $G$ is amenable and that $X$ is a standard Borel $G$-space. By Connes-Feldman-Weiss [3], if $\mu$ is any Borel probability measure on $X$, then there exists a $G$-invariant Borel subset $X_{0} \subseteq X$ with $\mu\left(X_{0}\right)=1$ such that the restriction $E_{G}^{X} \uparrow X_{0}=E_{G}^{X} \cap\left(X_{0} \times X_{0}\right)$ is hyperfinite and it follows that $E_{G}^{X} \uparrow X_{0} \leq_{B} E(G, 2)$. While these considerations do not rule out the existence of an amenable group $G$ such that $E(G, 2)$ is not $G$-universal, they suggest that it would be more effective to focus our attention on non-amenable groups with no nonabelian free subgroups. In Section 6, we will prove the following result.

Theorem 1.7. If $G$ is a simple quasi-finite sofic Kazhdan group, then

$$
E(G, 2)<_{B} E(G, 3)<_{B} \cdots<_{B} E(G, n)<_{B} \cdots<_{B} E(G, \mathbb{N}) .
$$

It is currently not known whether there are any groups satisfying the hypotheses of Theorem 1.7. However, if every hyperbolic group is residually finite, then such groups exist. (For more on the question of the residual finiteness of hyperbolic groups, see Kapovich-Wise [11].)

This paper is organized as follows. In Section 2, we will recall some basic notions from the theory of countable Borel equivalence relations; and we will state an easily applicable consequence of Popa's Cocycle Superrigidity Theorem which does not explicitly mention Borel cocycles. In Section 3, we will introduce the notion of a weakly action universal group; and we will prove that if $G$ is weakly action universal, then the conjugacy relation $\approx G$ of $G$ on the space of its subgroups is not essentially free. In Section 4, we will consider the question of which countable Borel equivalence relations can be realized up to Borel bireducibility as $\approx_{G}$ for some countable group $G$; and we will prove that there exists an uncountable family $\left\{G_{\alpha} \mid \alpha<2^{\aleph_{0}}\right\}$ of groups such that the conjugacy relations $\approx_{G_{\alpha}}$ are pairwise incomparable with respect to Borel reducibility. In Section 5, we will prove that if $n$ is a sufficiently large odd integer and $G=B(2, n)$ is the free 2-generator Burnside group of exponent $n$, then $E(G, 2)$ is not essentially free. Finally, in Section 6 , we will switch our attention from universal actions to $G$-universal actions; and we will use the remarkable recent work of Bowen [2] on the ergodic theory of sofic groups to prove Theorem 1.7.

\section{Preliminaries}

In this section, we will recall some basic notions from the theory of countable Borel equivalence relations; and we will state an easily applicable consequence of Popa's Cocycle Superrigidity Theorem which does not explicitly mention Borel cocycles. 
2.1. Countable Borel equivalence relations. A detailed development of the general theory of countable Borel equivalence relations can be found in Jackson-KechrisLouveau [10]. Here we will only remind the reader of a few basic notions.

Suppose that $E, F$ are countable Borel equivalence relations on the standard Borel spaces $X, Y$ respectively. Then a Borel map $\varphi: X \rightarrow Y$ is said to be a homomorphism from $E$ to $F$ if for all $x, y \in X$,

$$
x E y \Longrightarrow \varphi(x) F \varphi(y) .
$$

If $\varphi$ satisfies the stronger property that for all $x, y \in X$,

$$
x E y \Longleftrightarrow \varphi(x) F \varphi(y),
$$

then $\varphi$ is said to be a Borel reduction and we write $E \leq_{B} F$. If both $E \leq_{B} F$ and $F \leq_{B} E$, then we write $E \sim_{B} F$ and say that $E, F$ are Borel bireducible. We write $E<_{B} \quad F$ if both $E \leq_{B} F$ and $E \bigsqcup_{B} F$. Finally, if there exists a countable-toone Borel homomorphism $\varphi: X \rightarrow Y$ from $E$ to $F$, then we say that $E$ is weakly Borel reducible to $F$ and write $E \leq_{B}^{w} F$. In this case, $\varphi$ is said to be a weak Borel reduction from $E$ to $F$. As expected, a countable Borel equivalence relation $E$ is weakly universal if $F \leq_{B}^{w} E$ for every countable Borel equivalence relation $F$. (It is currently not known whether there exists a weakly universal countable Borel equivalence relation which is not universal. For a discussion of this interesting open problem, see Thomas [29].)

Suppose that $G$ is a countable group and that $X$ is a standard Borel $G$-space; i.e. that there exists a Borel action $(g, x) \mapsto g \cdot x$ of $G$ on $X$. Then $G$ is said to act freely on $X$ if $g \cdot x \neq x$ for all $1 \neq g \in G$ and $x \in X$. In this case, we say that $X$ is a free standard Borel $G$-space. If $E$ is a countable Borel equivalence on the standard Borel space $X$, then $E$ is said to be free if there exists a countable group $G$ with a free Borel action on $X$ such that $E_{G}^{X}=E$. The countable Borel equivalence relation $E$ is said to be essentially free if there exists a free countable Borel equivalence relation $F$ such that $E \sim_{B} F$.

2.2. Popa Superrigidity. The proofs of most of the results in this paper make essential use of Popa's Cocycle Superrigidity Theorem [24]. In this subsection, in order to make the paper intelligible to readers who are unfamiliar with the notions and techniques of superrigidity theory, we will state an easily applicable consequence of Popa's Theorem which does not explicitly mention Borel cocycles. First we need to give two preliminary definitions.

Definition 2.1. Suppose that $E, F$ are countable Borel equivalence relations on the standard Borel spaces $X, Y$ and that $\mu$ is a Borel probability measure on $X$. Then the Borel homomorphism $\varphi: X \rightarrow Y$ from $E$ to $F$ is said to be $\mu$-trivial if there exists a Borel subset $Z \subseteq X$ with $\mu(Z)=1$ such that $\varphi$ maps $Z$ into a single $F$-class. Otherwise, $\varphi$ is said to be $\mu$-nontrivial. 
Definition 2.2. If $G, H$ are groups, then the group homomorphism $\pi: G \rightarrow H$ is a virtual embedding if the kernel ker $\pi$ is finite.

Throughout this paper, $\mu_{m}$ will denote the usual product probability measure on $m^{G}$. The following result is an easy consequence of Popa's Cocycle Superrigidity Theorem [24]. (For example, see Thomas [28, Section 5].) Here we will only mention that the hypothesis that $Z$ is a free standard Borel $H$-space is necessary in order to be able to define a cocycle to which Popa's Theorem can be applied.

Theorem 2.3. Let $\Gamma$ be a countably infinite Kazhdan group and let $G$ be a countable group such that $\Gamma \unlhd G$. Suppose that $H$ is any countable group and that $Z$ is a free standard Borel $H$-space. If $\varphi: m^{G} \rightarrow Z$ is a $\mu_{m}$-nontrivial Borel homomorphism from $E(G, m)$ to $E_{H}^{Z}$, then there exists

(i) a virtual embedding $\pi: G \rightarrow H$,

(ii) a $G$-invariant Borel subset $Y \subseteq m^{G}$ with $\mu_{m}(Y)=1$, and

(iii) a Borel map $b: m^{G} \rightarrow H$

such that the "adjusted homomorphism" $\varphi^{\prime}(y)=b(y) \varphi(y)$ satisfies

$$
\varphi^{\prime}(g \cdot y)=\pi(g) \cdot \varphi^{\prime}(y)
$$

for all $g \in G$ and $y \in Y$.

\section{Weakly action universal groups}

In this section, we will introduce the notion of a weakly action universal group; and we will prove that if $G$ is weakly action universal, then the conjugacy relation of $G$ on the space of its subgroups is necessarily complicated. (As we will see, the appropriate level of generality for our study turns out to be the class of weakly action universal groups rather than the more obvious class of action universal groups.) Recall that a countable Borel equivalence relation $E$ is weakly universal if for every countable Borel equivalence relation $F$, there exists a weak Borel reduction (i.e. a countableto-one Borel homomorphism) from $F$ to $E$.

Definition 3.1. A countable group $G$ is said to be weakly action universal if there exists a standard Borel $G$-space $X$ such that $E_{G}^{X}$ is weakly universal.

The following basic result will play a key role in the remainder of this paper. (It should be pointed out that Theorem 3.2 is an easy consequence of Popa's Cocycle Superrigidity Theorem [24].)

Theorem 3.2 (Thomas [28]). Suppose that $G$ is a countable group and that $X$ is a standard Borel $G$-space. If $E_{G}^{X}$ is weakly universal, then $E_{G}^{X}$ is not essentially free. 
Thus if $E_{G}^{X}$ is weakly universal, then there necessarily exist many $x \in X$ such that the point stabilizer $G_{x}=\{g \in G \mid g \cdot x=x\}$ is nontrivial. Furthermore, recall that if $g \cdot x=y$, then $G_{y}=g G_{x} g^{-1}$. This suggests that we should study the complexity of the conjugacy relation of $G$ on the space of its subgroups.

Definition 3.3. If $G$ is a countable group, then $\operatorname{Sg}(G)$ denotes the standard Borel space of the subgroups of $G$ and $\approx_{G}$ denotes the conjugacy relation on $\operatorname{Sg}(G)$, which is defined by

$$
K \approx_{G} L \Longleftrightarrow(\exists g \in G) L=g K g^{-1}
$$

If Martin's Conjecture on Turing degree invariant Borel maps is true, then we have the following characterization of the class of weakly action universal groups. However, it should be pointed out that Martin's Conjecture has been an open problem for over 30 years.

Theorem 3.4. Assuming Martin's Conjecture, if $G$ is a countable group, then the following are equivalent:

(i) $G$ is weakly action universal.

(ii) $\approx_{G}$ is weakly universal.

Before we can state Martin's Conjecture, we must first recall some basic notions from recursion theory. We will follow the usual convention of identifying the powerset $\mathcal{P}(\mathbb{N})$ of the natural numbers with the Cantor space $2^{\mathbb{N}}$, by identifying each subset $A \in \mathcal{P}(\mathbb{N})$ with its characteristic function $\chi_{A} \in 2^{\mathbb{N}}$. If $A, B \in 2^{\mathbb{N}}$, then $B$ is Turing reducible to $A$, written $B \leq_{T} A$, if there exists an oracle Turing machine which computes $\chi_{B}$ when its oracle tape contains $\chi_{A}$. Here an oracle Turing machine is a Turing machine with a second "read only" tape, called the oracle tape, upon which we can write the characteristic function of any set $A \in 2^{\mathbb{N}}$, which is called the oracle. (For more details, see Rogers [25].) The Turing equivalence relation $\equiv_{T}$ on $2^{\mathbb{N}}$ is defined by

$$
A \equiv{ }_{T} B \Longleftrightarrow A \leq_{T} B \text { and } B \leq_{T} A .
$$

Finally for each $A \in 2^{\mathbb{N}}$, the corresponding cone is $\ell=\left\{B \in 2^{\mathbb{N}} \mid A \leq T \quad B\right\}$. (When studying the Turing equivalence relation, the set of cones plays an analogous role to that played by the full-measure subsets in ergodic theory.)

By Martin's Conjecture, we mean the following special case of a more general conjecture (also known as the 5th Victoria Delfino Problem) which was formulated by Martin in Kechris-Moschovakis [13].

Martin's Conjecture. If $\varphi: 2^{\mathbb{N}} \rightarrow 2^{\mathbb{N}}$ is a Borel homomorphism from $\equiv_{T}$ to $\equiv_{T}$, then exactly one of the following conditions holds:

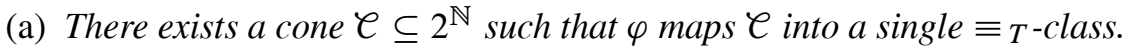


(b) There exists a cone $\mathcal{C} \subseteq 2^{\mathbb{N}}$ such that $x \leq_{T} \varphi(x)$ for all $x \in \mathcal{C}$.

Proof of Theorem 3.4. It is clear that (ii) implies (i). Conversely, suppose that $X$ is a standard Borel $G$-space such that $E_{G}^{X}$ is weakly universal and suppose that $\approx_{G}$ is not weakly universal. Consider the Borel map $\varphi: X \rightarrow \operatorname{Sg}(G)$ defined by

$$
\varphi(x)=G_{x}=\{g \in G \mid g \cdot x=x\} .
$$

Then $\varphi$ is a Borel homomorphism from $E_{G}^{X}$ to $\approx_{G}$. Let $\psi: 2^{\mathbb{N}} \rightarrow X$ be a weak Borel reduction from $\equiv{ }_{T}$ to $E_{G}^{X}$ and let $\theta=\varphi \circ \psi$. Assuming Martin's Conjecture, by Thomas [29, Theorem 1.4], since $\theta$ is a Borel homomorphism from $\equiv_{T}$ to $\approx_{G}$ and $\approx_{G}$ is not weakly universal, there exists a cone $\mathcal{C} \subseteq 2^{\mathbb{N}}$ such that $\theta$ maps $\mathcal{C}$ into a single $\approx_{G}$-class. (Here it should be emphasized that the currently known "proof" of Thomas [29, Theorem 1.4], makes essential use of Martin's Conjecture.) Hence, after slightly adjusting $\psi$ if necessary, we can suppose that there exists a fixed subgroup $K \leqslant G$ such that $G_{\psi(A)}=K$ for all $A \in \mathcal{C}$. For later use, note that $\equiv_{T} \uparrow \mathcal{C}$ is weakly universal. (For example, see Thomas [29, Observation 2.3].)

Let $X_{0}=\left\{x \in X \mid G_{x}=K\right\}$. If $x, y \in X_{0}$ and $x E_{G}^{X} y$, then there exists an element $g \in G$ such that $g \cdot x=y$. Since

$$
g K g^{-1}=g G_{x} g^{-1}=G_{y}=K,
$$

it follows that $g \in N_{G}(K)$. Furthermore, if $g^{\prime} \in N_{G}(K)$ also satisfies $g^{\prime} \cdot x=y$, then $g^{\prime} K=g K$. Thus $E_{G}^{X} \uparrow X_{0}$ can be realized as the orbit equivalence relation of the corresponding free Borel action of $\Delta=N_{G}(K) / K$ on $X_{0}$. Applying Theorem 3.2, it follows that $E_{G}^{X} \uparrow X_{0}$ is not weakly universal. But this is a contradiction, since $\equiv_{T} \uparrow \mathcal{C} \leq_{B}^{w} E_{G}^{X} \uparrow X_{0}$ and $\equiv_{T} \uparrow \mathcal{C}$ is weakly universal.

Unfortunately, as we mentioned earlier, it is currently not known whether Martin's Conjecture is true. In the remainder of this section, we will prove the following weak version of Theorem 3.4.

Theorem 3.5. If $\approx_{G}$ is essentially free, then $G$ is not weakly action universal.

Question 3.6. Does the converse of Theorem 3.5 hold?

The proof of Theorem 3.5 makes use of Popa Superrigidity, together with the following result.

Theorem 3.7 (Andretta-Camerlo-Hjorth [1]). If the countable group $G$ has a free nonabelian subgroup, then $\approx_{G}$ is countable universal.

Proof of Theorem 3.5. Suppose that $G$ is a weakly action universal group such that $\approx_{G}$ is essentially free. Then there exists a countable group $H$ and a free standard Borel $H$-space $Z$ such that $\approx_{G} \sim_{B} E_{H}^{Z}$. Let $\varphi: \operatorname{Sg}(G) \rightarrow Z$ be a Borel reduction 
from $\approx_{G}$ to $E_{H}^{Z}$. For later use, let $L$ be a finitely generated group with no nontrivial finite normal subgroups such that $L$ does not embed into $H$. (To see that such a group $L$ exists, recall that there exist uncountably many finitely generated groups up to isomorphism and that $H$ has only countably many finitely generated subgroups. Hence there exists a finitely generated group $L_{0}$ which does not embed into $H$ and we can let $L$ be the free product $\mathbb{Z} * L_{0}$.) Let $\Gamma=\operatorname{SL}_{3}(\mathbb{Z}) \times L$ and note that $\Gamma$ also has no nontrivial finite normal subgroups.

Next let $X$ be a standard Borel $G$-space such that $E_{G}^{X}$ is weakly universal and let $\psi: 2^{\Gamma} \rightarrow X$ be a weak Borel reduction from $E(\Gamma, 2)$ to $E_{G}^{X}$. Let $\sigma: X \rightarrow \operatorname{Sg}(G)$ be the Borel homomorphism defined by $\sigma(x)=G_{x}$ and let $\theta: 2^{\Gamma} \rightarrow Z$ be the Borel homomorphism from $E(\Gamma, 2)$ to $E_{H}^{Z}$ defined by $\theta=\varphi \circ \sigma \circ \psi$. Applying Theorem 2.3, since $\Gamma$ does not embed into $H$, there exists a Borel subset $Y \subseteq 2^{\Gamma}$ with $\mu_{2}(Y)=1$ such that $\theta$ maps $Y$ into a single $E_{H}^{Z}$-class; and this implies that $\sigma \circ \psi$ maps $Y$ into a single conjugacy class of subgroups of $G$. Hence, after slightly adjusting $\psi$ if necessary, we can suppose that there exists a fixed subgroup $K \leqslant G$ such that $G_{\psi(y)}=K$ for all $y \in Y$. Let $X_{0}=\left\{x \in X \mid G_{x}=K\right\}$. Then $E_{G}^{X} \uparrow X_{0}$ can be realized as the orbit equivalence relation of the corresponding free Borel action of the quotient group $\Delta=N_{G}(K) / K$. Clearly the weak Borel reduction $\psi \uparrow Y$ from $E(\Gamma, 2) \uparrow Y$ to $E_{G}^{X} \uparrow X_{0}$ can be extended to a $\mu_{2}$-nontrivial Borel homomorphism from $E(\Gamma, 2)$ to $E_{G}^{X} \uparrow X_{0}$. Hence, by Theorem 2.3, there exists an embedding $\pi: \Gamma \rightarrow \Delta$. In particular, since $\mathrm{SL}_{3}(\mathbb{Z}) \leqslant \Gamma$, it follows that $\Delta=N_{G}(K) / K$ has a nonabelian free subgroup; and this implies that $G$ also has a nonabelian free subgroup. Applying Theorem 3.7, it follows that $\approx_{G}$ is countable universal and hence $\approx_{G}$ is not essentially free, which is a contradiction.

\section{The conjugacy relation on the space of subgroups}

In this section, we will consider the question of which countable Borel equivalence relations can be realized up to Borel bireducibility as $\approx_{G}$ for some countable group $G$. As we mentioned earlier, Andretta-Camerlo-Hjorth [1] have shown that if $G$ has a free nonabelian subgroup, then $\approx_{G}$ is countable universal. On the other hand, by Jackson-Kechris-Louveau [10, Proposition 2.13], if $G$ is amenable, then $\approx_{G}$ is Fréchet amenable. (For the definition of Fréchet amenability, see Jackson-KechrisLouveau [10, Section 2.4]. It is currently not known whether every Fréchet amenable countable Borel equivalence relation is hyperfinite.) The main result of this section provides many examples of groups $G$ such that $\approx_{G}$ is neither Fréchet amenable nor countable universal. However, we should point out that the following fundamental question remains open.

Question 4.1. Suppose that $E$ is any countable Borel equivalence relation. Does there necessarily exist a countable group $G$ such that $\approx_{G} \sim_{B} E$ ? 
We will begin with some basic observations concerning the relative complexity of the conjugacy relations $\approx_{G}, \approx_{H}$ for various pairs $G, H$ of groups.

Lemma 4.2. If $G, H$ are countable groups and there exists a surjective homomorphism $\pi: G \rightarrow H$, then $\approx_{H} \leq_{B} \approx_{G}$.

Proof. Let $\varphi: \operatorname{Sg}(H) \rightarrow \operatorname{Sg}(G)$ be the map defined by $\varphi(K)=\pi^{-1}(K)$. Then $\varphi$ is a Borel reduction from $\approx_{H}$ to $\approx_{G}$.

Next recall that a subgroup $H$ of a group $G$ is said to be malnormal if whenever $g \in G \backslash H$, then $g H g^{-1} \cap H=1$.

Lemma 4.3. If $H$ is a malnormal subgroup of $G$, then $\approx_{H} \leq_{B} \approx_{G}$.

Proof. The inclusion map $\operatorname{Sg}(H) \hookrightarrow \operatorname{Sg}(G)$ is a Borel reduction from $\approx_{H}$ to $\approx_{G}$.

Question 4.4. Do there exist countable groups $H \leqslant G$ such that $\approx_{H} \not_{B} \approx_{G}$ ?

Remark 4.5. By Theorem 3.7, if $H \leqslant G$ is such a pair, then $G$ has no nonabelian free subgroups.

Finally if $H, C$ are any groups, then $C$ wr $H$ denotes the (restricted) wreath product of $H$ and $C$, which is defined as follows. For each function $f: H \rightarrow C$, the support $\sigma(f)$ is defined to be

$$
\sigma(f)=\{x \in H \mid f(x) \neq 1\}
$$

and the corresponding base group is defined to be

$$
B=\{f: H \rightarrow C \mid \sigma(f) \text { is finite }\},
$$

equipped with pointwise multiplication; i.e., if $f, g \in B$, then

$$
(f g)(x)=f(x) g(x)
$$

for all $x \in H$. There is a natural action of $H$ on $B$ defined by

$$
(a \cdot f)(x)=f\left(a^{-1} x\right)
$$

for all $f \in B$ and $a, x \in H$; and $C$ wr $H$ is the corresponding semidirect product $B \rtimes H$. For each $x \in H$, let

$$
C_{x}=\{f \in B \mid f(y)=1 \text { for all } x \neq y \in H\} .
$$

Then $B=\bigoplus_{x \in H} C_{x}$; and $a C_{x} a^{-1}=C_{a x}$ for all $a, x \in H$. 
Lemma 4.6. If $H, C$ are countable groups and $G=C$ wr $H$ is the corresponding (restricted) wreath product, then $E(H, 2) \leq_{B} \approx_{G}$.

Proof. Let $B=\bigoplus_{x \in H} C_{x}$ be the base group of $G=C$ wr $H$; and for each subset $A \subseteq H$, let $K_{A}$ be the subgroup of $B$ defined by $K_{A}=\bigoplus_{a \in A} C_{a}$. Suppose that $g=h b \in G$ is any element, where $h \in H$ and $b \in B$. Since $K_{A} \unlhd B$, we have that

$$
g K_{A} g^{-1}=h b K_{A} b^{-1} h^{-1}=h K_{A} h^{-1}=K_{h A} .
$$

It follows that the map $A \mapsto K_{A}$ is a Borel reduction from $E(H, 2)$ to $\approx_{G}$.

The remainder of this section will be devoted to the proof of the following result.

Theorem 4.7. There exists an uncountable family $\left\{G_{\alpha} \mid \alpha<2^{\aleph_{0}}\right\}$ of countable groups such that for all $\alpha<\beta<2^{\aleph_{0}}$,

(i) $\approx_{G_{\alpha}}$ is essentially free;

(ii) $\approx_{G_{\alpha}}$ is not Fréchet amenable; and

(ii) $\approx_{G_{\alpha}}$ and $\approx_{G_{\beta}}$ are incomparable with respect to Borel reducibility.

Remark 4.8. Note that each $G_{\alpha}$ satisfies the requirements of Theorem 1.6. To see this, first notice that since $\approx_{G_{\alpha}}$ is not Fréchet amenable, it follows that $G$ is not amenable. (Here we are applying Jackson-Kechris-Louveau [10, Proposition 2.13].) Secondly, by Theorem 3.5 , since $\approx_{G_{\alpha}}$ is essentially free, it follows that $G_{\alpha}$ is not weakly action universal.

Each $G_{\alpha}$ will have the form $C_{2}$ wr $\Gamma_{\alpha}$, where $C_{2}$ is the cyclic group of order 2 and $\Gamma_{\alpha}$ is a suitably chosen simple quasi-finite group. Here an infinite group $\Gamma$ is said to be quasi-finite if every proper subgroup of $\Gamma$ is finite. It is easily shown that every abelian quasi-finite group is isomorphic to a quasi-cyclic group $C_{p} \infty$ for some prime $p$. (See Ol'shanskii [17, Theorem 7.5].) However, it was a long outstanding problem whether there existed a nonabelian quasi-finite group. This problem was finally solved by Ol'shanskii in his celebrated papers [14], [15]. A clear account of this work can be found in Ol'shanskii [17]. The following result will play an essential role in the proofs of both Theorem 4.7 and Theorem 1.7.

Proposition 4.9 (Thomas [28]). Suppose that $\Gamma$ is a simple quasi-finite group and that $X$ is a standard Borel $\Gamma$-space. If $Z=\left\{x \in X \mid \Gamma_{x}=1\right\}$ is the free part of the action, then $E_{\Gamma}^{X} \sim_{B} E_{\Gamma}^{X} \uparrow Z$.

The following result is implicitly contained in Ol'shanskii [19]. (For more details, see Ozawa [21].)

Theorem 4.10 (Ol'shanskii [19]). If $H$ is a noncyclic torsion-free hyperbolic group, then $H$ has a family $\left\{\Gamma_{\alpha}=H / N_{\alpha} \mid \alpha<2^{\aleph_{0}}\right\}$ of uncountably many pairwise nonisomorphic simple quasi-finite quotient groups. 
Remark 4.11. Suppose that $H$ is a noncyclic torsion-free hyperbolic Kazhdan group. (For example, we can let $H$ be a co-compact lattice in $\operatorname{Sp}(n, 1)$ for some $n \geq 2$. See de la Harpe-Valette [9].) Then each $\Gamma_{\alpha}=H / N_{\alpha}$ is also a Kazhdan group and hence is non-amenable. Applying Proposition 4.9 and Theorem 3.2, it follows that $\Gamma_{\alpha}$ is not weakly action universal. Thus each $\Gamma_{\alpha}$ also satisfies the requirements of Theorem 1.6. Of course, since $\Gamma_{\alpha}$ is quasi-finite, it follows that $\operatorname{Sg}\left(\Gamma_{\alpha}\right)$ is countable and hence $\approx_{\Gamma_{\alpha}}$ is smooth.

Proof of Theorem 4.7. Let $H$ be a noncyclic torsion-free hyperbolic Kazhdan group and let $\left\{\Gamma_{\alpha}=H / N_{\alpha} \mid \alpha<2^{\aleph_{0}}\right\}$ be a family of uncountably many pairwise nonisomorphic simple quasi-finite quotient groups. For each $\alpha<2^{\aleph_{0}}$, let $G_{\alpha}=$ $C_{2}$ wr $\Gamma_{\alpha}$, where $C_{2}$ is the cyclic group of order 2 .

To see that each $\approx_{G_{\alpha}}$ is not Fréchet amenable, first notice that each $\Gamma_{\alpha}$ is an infinite Kazhdan group and thus is non-amenable. Hence, by Jackson-KechrisLouveau [10, Proposition 2.14], $E\left(\Gamma_{\alpha}, 2\right)$ is not Fréchet amenable. By Lemma 4.6, $E\left(\Gamma_{\alpha}, 2\right) \leq_{B} \approx_{G_{\alpha}}$ and this implies that $\approx_{G_{\alpha}}$ is not Fréchet amenable.

Next we will show that each $\approx_{G_{\alpha}}$ is essentially free. Fix some $\alpha<2^{\aleph_{0}}$. In order to simplify notation, let $G=G_{\alpha}$ and $\Gamma=\Gamma_{\alpha}$. Let $\pi: G \rightarrow \Gamma$ be the canonical surjective homomorphism. Then $\operatorname{Sg}(G)=X \sqcup Y \sqcup Z$, where

- $X=\{H \leqslant G \mid \pi[H]=\Gamma\}$;

- $Y=\{H \leqslant G \mid \pi[H]$ is a nontrivial finite subgroup of $\Gamma\}$; and

- $Z=\{H \leqslant G \mid \pi[H]=1\}$.

We will successively analyze the Borel complexity of $\approx_{G}$ restricted to each of the above Borel subsets of $\operatorname{Sg}(G)$. From now on, let $B=\bigoplus_{x \in \Gamma} C_{x}$ be the base group of $G=C_{2}$ wr $\Gamma$, so that $G=B \rtimes \Gamma$.

Claim 4.12. $\approx_{G} \uparrow X$ is smooth.

Proof of Claim 4.12. Suppose that $H \in X$ and let $g=\gamma b \in G$ be any element, where $\gamma \in \Gamma$ and $b \in B$. Since $\pi[H]=\Gamma$, there exists an element $c \in B$ such that $h=\gamma c \in H$. It follows that

$$
g(H \cap B) g^{-1}=\gamma(H \cap B) \gamma^{-1}=h(H \cap B) h^{-1}=H \cap B .
$$

Thus $H \cap B \unlhd G$. Also notice that since $H / H \cap B \cong \Gamma$, it follows that $H$ is finitely generated over $H \cap B$. Hence there exist only countably many $H^{\prime} \in X$ such that $H^{\prime} \cap B=H \cap B$. It follows that if $\equiv$ is the equivalence relation defined on $X$ by

$$
H \equiv H^{\prime} \Longleftrightarrow H \cap B=H^{\prime} \cap B,
$$

then $\equiv$ is a smooth countable Borel equivalence relation. Since $\approx_{G} \uparrow X \subseteq \equiv$, it follows that $\approx_{G} \uparrow X$ is also smooth. (For example, see Thomas [30, Lemma 2.1].) 
Claim 4.13. $\approx_{G} \uparrow Y$ is smooth.

Proof of Claim 4.13. Let $\mathcal{F}$ be a set of representatives of the countably many conjugacy classes of nontrivial finite subgroups of $\Gamma$; and for each $F \in \mathcal{F}$, let

$$
Y_{F}=\{H \in Y \mid \pi[H]=F\} .
$$

Then clearly $\approx_{G} \uparrow Y$ is Borel bireducible with $\approx_{G} \uparrow \bigsqcup_{F \in \mathscr{F}} Y_{F}$. Hence it is enough to prove that each $\approx_{G} \uparrow Y_{F}$ is smooth. Fix some $F \in \mathscr{F}$ and suppose that $H \in Y_{F}$. Since $H / H \cap B \cong F$ is finite, it follows that there exist only countably many $H^{\prime} \in Y_{F}$ such that $H^{\prime} \cap B=H \cap B$. Hence if $\sim$ is the equivalence relation on $Y_{F}$ defined by

$$
H \sim H^{\prime} \Longleftrightarrow\left(\exists \gamma \in N_{\Gamma}(F)\right) \gamma(H \cap B) \gamma^{-1}=H^{\prime} \cap B,
$$

then $\sim$ is a countable Borel equivalence relation. Since $\Gamma$ is a simple quasi-finite group, it follows that $N_{\Gamma}(F)$ is a finite subgroup of $\Gamma$ and hence $\sim$ is smooth. Thus it is enough to show that $\approx_{G} \uparrow Y_{F} \subseteq \sim$. To see this, let $H, H^{\prime} \in Y_{F}$ and suppose that $\mathrm{gHg}^{-1}=H^{\prime}$. Let $g=\gamma b$, where $\gamma \in \Gamma$ and $b \in B$. Then clearly $\gamma \in N_{\Gamma}(F)$ and since

$$
H^{\prime} \cap B=g(H \cap B) g^{-1}=\gamma(H \cap B) \gamma^{-1},
$$

it follows that $H \sim H^{\prime}$.

Using Claim 4.12 and Claim 4.13, it follows that $\approx_{G}$ is Borel bireducible with $\approx_{G} \uparrow Z$ and thus it only remains to analyze the Borel complexity of $\approx_{G} \uparrow Z$. Suppose that $H \in Z$. Let $g=\gamma b \in G$ be any element, where $\gamma \in \Gamma$ and $b \in B$. Since $H \leqslant B$, it follows that $g \mathrm{Hg}^{-1}=\gamma H \gamma^{-1}$. Thus $\approx_{G} \uparrow Z$ is the orbit equivalence relation induced by the conjugacy action of the simple quasi-finite $\Gamma$; and applying Proposition 4.9, it follows that $\approx_{G} \uparrow Z$ is essentially free. This completes the proof that $\approx_{G_{\alpha}}$ is essentially free.

Finally we will prove that if $\alpha \neq \beta$, then $\approx_{G_{\alpha}}$ and $\approx_{G_{\beta}}$ are incomparable with respect to Borel reducibility. Suppose that $\approx_{G_{\alpha}} \leq_{B} \approx_{G_{\beta}}$. By Lemma 4.6, we have that $E\left(\Gamma_{\alpha}, 2\right) \leq_{B} \approx_{G_{\alpha}}$ and hence $E\left(\Gamma_{\alpha}, 2\right) \leq_{B} \approx_{G_{\beta}}$. Furthermore, combining Proposition 4.9 with the argument in the previous paragraph, it follows that there exists a free standard Borel $\Gamma_{\beta}$-space $Z^{\prime}$ such that $E_{\Gamma_{\beta}}^{Z^{\prime}} \sim_{B} \approx_{G_{\beta}}$. Let $\varphi: 2^{\Gamma_{\alpha}} \rightarrow Z^{\prime}$ be a Borel reduction from $E\left(\Gamma_{\alpha}, 2\right)$ to $E_{\Gamma_{\beta}}^{Z^{\prime}}$. Applying Theorem 2.3, there exists an embedding $\theta: \Gamma_{\alpha} \rightarrow \Gamma_{\beta}$; and since $\Gamma_{\beta}$ is quasi-finite, it follows that $\theta$ is an isomorphism, which is a contradiction. This completes the proof of Theorem 4.7.

\section{Free Burnside groups}

In this section, we will present some evidence which supports the conjecture that free Burnside groups of sufficiently large odd exponent are action universal. The 
following result implies that this conjecture is equivalent to the statement that if $n$ is a sufficiently large odd integer, then there exists an action universal group $H$ of exponent $n$.

Proposition 5.1. Let $n$ be a sufficiently large odd integer and let $2 \leq m \leq \omega$. If $G=B(m, n)$ is the free m-generator Burnside group of exponent $n$ and $H$ is any countable group of exponent $n$, then:

(i) $\approx_{H} \leq_{B} \approx_{G}$; and

(ii) if $X$ is any standard Borel space, then $E(H, X) \leq_{B} E(G, X)$.

Proof. If $K=B(\omega, n)$ is the free Burnside group of exponent $n$ on countably many generators, then there exists a surjective homomorphism $\varphi: K \rightarrow H$. Hence, by Lemma 4.2, it follows that $\approx_{H} \leq_{B} \approx_{K}$. By Ol'shanskii-Sapir [20, Lemma 4.11] and Sonkin [26, Section 4], there exists a malnormal subgroup $L$ of $G$ such that $L \cong K$; and hence, applying Lemma 4.3, it follows that $\approx_{K} \leq_{B} \approx_{G}$. Thus $\approx_{H} \leq_{B} \approx_{G}$. Similarly, applying Dougherty-Jackson-Kechris [4, Section 1], it follows that if $X$ is any standard Borel space, then $E(H, X) \leq_{B} E(G, X)$.

Most of this section will be devoted to the proof of the following result.

Theorem 5.2. Let $n$ be a sufficiently large odd integer and let $2 \leq m \leq \omega$. If $G=B(m, n)$ is the free m-generator Burnside group of exponent $n$, then $E(G, 2)$ is not essentially free.

Before beginning the proof of Theorem 5.2, we first derive the following easy consequence.

Corollary 5.3. Suppose that $n$ is a sufficiently large odd composite integer and that $2 \leq m \leq \omega$. If $G=B(m, n)$ is the free m-generator Burnside group of exponent $n$, then $\approx_{G}$ is not essentially free.

Proof. By Proposition 5.1, it is enough to prove that if $n$ is a sufficiently large odd composite number and $G=B(3, n)$ is the free 3-generator Burnside group of exponent $n$, then $\approx_{G}$ is not essentially free. Let $p$ be a prime factor of $n$, chosen so that $n_{0}=n / p$ is still sufficiently large. Let $W=C_{p} \mathrm{wr} B\left(2, n_{0}\right)$. Then $W$ is a 3-generator group of exponent $n$ and hence there exists a surjective homomorphism $G \rightarrow W$. It follows that $\approx_{W} \leq_{B} \approx_{G}$. Also, by Lemma 4.6, we have that $E\left(B\left(2, n_{0}\right), 2\right) \leq \approx_{W}$. Hence Theorem 5.2 implies that $\approx_{G}$ is not essentially free.

Of course, Corollary 5.3 should also be true if $n$ is a sufficiently large prime. The following result will be used in the proof of Theorem 5.2.

Theorem 5.4 (Ol'shanskii [18]). If $H$ is a noncyclic torsion-free hyperbolic group, then there exists a natural number $n_{H}$ such that the group $H / H^{n}$ is infinite for every odd $n \geq n_{H}$. 
We will also make use of the following result of Ol'shanskii [17, Theorem 28.7]. (The groups given by Theorem 4.10 have infinite exponent and hence cannot be used in the proof of Theorem 5.2.)

Theorem 5.5 (Ol'shanskii [17]). For every sufficiently large odd integer $n$, there exists a family $\left\{G_{\alpha} \mid \alpha<2^{\aleph_{0}}\right\}$ of pairwise nonisomorphic infinite simple groups such that for each $\alpha<2^{\aleph_{0}}$,

(a) $G_{\alpha}$ is a group of exponent $n$; and

(b) every nontrivial proper subgroup of $G_{\alpha}$ is cyclic of order dividing $n$.

Remark 5.6. While Ol'shanskii does not state explicitly that each $G_{\alpha}$ is simple, this follows easily from the fact that the centralizer of each nontrivial element of $G_{\alpha}$ is cyclic. (See Ol'shanskii [17, Theorem 26.5].) For suppose that $N$ is a nontrivial proper normal subgroup of $G_{\alpha}$. Then $N$ is clearly finite; and by considering the action of $G_{\alpha}$ on $N$ via conjugation, it follows that $N$ must be contained in the center of $G_{\alpha}$, which is a contradiction.

Proof of Theorem 5.2. Let $H$ be a noncyclic torsion-free hyperbolic Kazhdan group. By Theorem 5.4, we can suppose that the group $K=H / H^{n}$ is infinite. Thus $K$ is an infinite Kazhdan group of exponent $n$. Let $\left\{G_{\alpha} \mid \alpha<2^{\aleph_{0}}\right\}$ be the family of pairwise nonisomorphic simple groups of exponent $n$ given by Theorem 5.5. Of course, it is clear that each $G_{\alpha}$ is a 2-generator group.

Let $K$ be a $d$-generator group. By Ol'shanskii [17, Theorem 39.1], $B(2, n)$ contains a subgroup which is isomorphic to the free Burnside group $B(d+2, n)$ on $d+2$ generators. Hence, by Dougherty-Jackson-Kechris [4, Proposition 1.5], we have that $E(B(d+2, n), 2) \leq_{B} E(B(2, n), 2)$ and so it is enough to show that $E(B(d+2, n), 2)$ is not essentially free. To see this, first notice that for each $\alpha<2^{\aleph_{0}}$, the group $L_{\alpha}=K \times G_{\alpha}$ is a homomorphic image of $B(d+2, n)$; and hence, by [4, Proposition 1.4], we have that $E\left(L_{\alpha}, 2\right) \leq_{B} E(B(d+2, n), 2)$. Now suppose that $E(B(d+2, n), 2) \sim_{B} E_{\Delta}^{X}$, where $\Delta$ is a countable group and $X$ is a free standard Borel $\Delta$-space. Then for each $\alpha<2^{\aleph_{0}}$, there exists a Borel reduction $\varphi_{\alpha}: 2^{L_{\alpha}} \rightarrow X$ from $E\left(L_{\alpha}, 2\right)$ to $E_{\Delta}^{X}$; and hence, by Theorem 2.3, there exists a virtual embedding $\pi_{\alpha}: L_{\alpha} \rightarrow \Delta$. Since $G_{\alpha}$ is an infinite simple group and ker $\pi_{\alpha}$ is finite, it follows that $\pi_{\alpha} \uparrow G_{\alpha}$ is an embedding. Since $\Delta$ has only countably many 2-generator subgroups, it follows that there exist $\alpha \neq \beta$ such that $\pi_{\alpha}\left[G_{\alpha}\right]=\pi_{\beta}\left[G_{\beta}\right]$, which contradicts the fact that $G_{\alpha}$ and $G_{\beta}$ are nonisomorphic.

\section{6. $G$-universal actions}

In this final section, we will switch our attention from universal actions to $G$-universal actions. Recall that Jackson-Kechris-Louveau [10, Theorem 5.4], have shown that if $G$ is any countable group, then $E(G, \mathbb{N})$ is $G$-universal. On the other hand, there are 
currently no countable groups $G$ for which it is known that $E(G, 2)$ is not $G$-universal. We will initially focus on the free parts of the various shift actions.

Definition 6.1. For each countable group $G$ and standard Borel space $X$, the free part of $X^{G}$ is

$$
(X)^{G}=\left\{p \in X^{G} \mid g \cdot p \neq p \text { for all } 1 \neq g \in G\right\} ;
$$

and $F(G, X)=E(G, X) \uparrow(X)^{G}$ is the corresponding orbit equivalence relation.

By Jackson-Kechris-Louveau [10, Section 5.1], if $G$ is any countable group and $Z$ is a free standard Borel $G$-space, then $E_{G}^{Z} \leq_{B} F(G, \mathbb{N})$. On the other hand, by Jackson-Kechris-Louveau [10, Theorem 3.17], letting $\mathbb{F}_{2}$ denote the free group on two generators, we have that $F\left(\mathbb{F}_{2}, 2\right) \sim_{B} F\left(\mathbb{F}_{2}, \mathbb{N}\right)$. The following is the main result of this section.

Theorem 6.2. If $G$ is an co-hopfian sofic Kazhdan group with no nontrivial finite normal subgroups, then

$$
F(G, 2)<_{B} F(G, 3)<_{B} \cdots<_{B} F(G, n)<_{B} \cdots<_{B} F(G, \mathbb{N}) .
$$

Here a group $G$ is said to be co-hopfian if every embedding $\pi: G \rightarrow G$ is an automorphism. A clear account of the basic theory of sofic groups can be found in Pestov [24]. It is an important open problem whether every group is sofic. For our purposes, it is enough to mention the following points:

- Every residually finite group is sofic; and, in particular, it follows that finitely generated linear groups are sofic. The results of Ol'shanskii [19] imply that if every hyperbolic group is residually finite, then there exist groups satisfying the hypotheses of Theorem 1.7.

- It is currently not known whether there exists an infinite finitely generated simple sofic group. ${ }^{1}$

Corollary 6.3. If $G=\mathrm{SL}_{3}(\mathbb{Z})$, then

$$
F(G, 2)<_{B} F(G, 3)<_{B} \cdots<_{B} F(G, n)<_{B} \cdots<_{B} F(G, \mathbb{N}) .
$$

Proof of Corollary 6.3. It is well known that $\mathrm{SL}_{3}(\mathbb{Z})$ is a Kazhdan group with no nontrivial finite normal subgroups; and by the preceding remarks, $\mathrm{SL}_{3}(\mathbb{Z})$ is sofic. Finally, by Steinberg [27, Theorem 6], $\mathrm{SL}_{3}(\mathbb{Z})$ is also co-hopfian.

Next we note that Theorem 1.7 is an easy consequence of Theorem 6.2.

\footnotetext{
${ }^{1}$ It has recently been proved that such groups do indeed exist. See Elek-Monod [5] or GrigorchukMedynets [8].
} 
Proof of Theorem 1.7. If $G$ is a simple quasi-finite sofic Kazhdan group, then $G$ clearly satisfies the hypotheses of Theorem 6.2; and by Proposition 4.9, we have that $E(G, X) \sim_{B} F(G, X)$ for every standard Borel space $X$.

The following notion will play a key role in the proof of Theorem 6.2.

Definition 6.4. Let $G$ be a countable group and let $Z$ be a standard Borel $G$-space. If $I$ is a countable set, then an $I$-generator is a partition $Z=\bigsqcup_{i \in I} A_{i}$ into Borel subsets such that $\left\{g \cdot A_{i} \mid i \in I, g \in G\right\}$ separates points. (Equivalently, the family of sets $\left\{g \cdot A_{i} \mid i \in I, g \in G\right\}$ generates the $\sigma$-algebra of Borel subsets of $Z$.)

Example 6.5. Consider the shift action of the countable group $G$ on the standard Borel space $Z=(m)^{G}$; and for each $i \in m$, let

$$
B_{i}=\left\{x \in(m)^{G} \mid x(1)=i\right\} .
$$

Then $\beta=\left(B_{0}, B_{1}, \ldots, B_{m-1}\right)$ is an $m$-generator.

Recall that if $G$ is a countable group and $m \geq 2$, then $\mu_{m}$ denotes the usual product probability measure on $m^{G}$. It is easily checked that $\mu_{m}\left((m)^{G}\right)=1$.

Lemma 6.6. If $G$ is a co-hopfian Kazhdan group with no nontrivial finite normal subgroups and $F(G, m) \leq_{B} F(G, n)$, then there exists a $G$-invariant Borel subset $Z \subseteq(m)^{G}$ with $\mu_{m}(Z)=1$ such that $Z$ admits an $n$-generator.

Proof. Suppose that $\varphi:(m)^{G} \rightarrow(n)^{G}$ is a Borel reduction from $F(G, m)$ to $F(G, n)$. Clearly $\varphi$ can be extended to a $\mu_{m}$-nontrivial Borel homomorphism from $E(G, m)$ to $F(G, n)$. Hence, by Theorem 2.3 , after slightly adjusting $\varphi$ if necessary, we can suppose that there exists a $G$-invariant Borel subset $Z \subseteq(m)^{G}$ with $\mu_{m}(Z)=1$ and an embedding $\pi: G \rightarrow G$ such that

$$
\varphi(g \cdot z)=\pi(g) \cdot \varphi(z)
$$

for all $g \in G$ and $z \in Z$. Since $G$ is co-hopfian, it follows that $\pi$ is an automorphism of $G$. Let $\beta=\left(B_{0}, B_{1}, \ldots, B_{n-1}\right)$ be the $n$-generator of $(n)^{G}$ given by Example 6.5; and for each $0 \leq i \leq n-1$, let $A_{i}=\varphi^{-1}\left(B_{i}\right) \cap Z$. Then we claim that $\alpha=$ $\left(A_{0}, A_{1}, \ldots, A_{n-1}\right)$ is an $n$-generator of $Z$. To see this, suppose that $y, z \in Z$ are distinct points. Clearly if $y, z$ lie in different $G$-orbits, then $\varphi(y) \neq \varphi(z)$. Otherwise, there exists $1 \neq g \in G$ such that $z=g \cdot y$ and $\operatorname{so} \varphi(z)=\pi(g) \cdot \varphi(y) \neq \varphi(y)$. Thus $\varphi(y) \neq \varphi(z)$ and hence there exists $0 \leq i \leq n-1$ and an element $g \in G$ such that $\varphi(y) \in g \cdot B_{i}$ and $\varphi(z) \notin g \cdot B_{i}$. Let $h=\pi^{-1}(g)$. Then $y \in h \cdot A_{i}$ and $z \notin h \cdot A_{i}$. Thus $\left\{h \cdot A_{i} \mid 0 \leq i \leq n-1, h \in G\right\}$ separates points.

Consequently, in order to prove Theorem 6.2, it is enough to rule out the existence of $n$-generators for full-measure subsets of $(m)^{G}$ for $n<m$. 
Definition 6.7. Suppose that $Z$ is a standard Borel space with Borel probability measure $\mu$ and $I$ is a countable set. If $Z=\bigsqcup_{i \in I} A_{i}$ is a partition into Borel subsets, then the entropy of $\alpha=\left(A_{i} \mid i \in I\right)$ is defined to be

$$
H(\alpha)=-\sum_{i \in I} \mu\left(A_{i}\right) \log \left(\mu\left(A_{i}\right)\right)
$$

Remark 6.8. If $A_{i}$ is a null set, then we define $\mu\left(A_{i}\right) \log \left(\mu\left(A_{i}\right)\right)=0$.

Example 6.9. Suppose that $Z=(m)^{G}$ and that $\beta=\left(B_{0}, B_{1}, \ldots, B_{m-1}\right)$ is the $m$-generator given in Example 6.5. Then

$$
H(\beta)=-\left(\frac{1}{m} \log \left(\frac{1}{m}\right)+\cdots+\frac{1}{m} \log \left(\frac{1}{m}\right)\right)=\log (m) .
$$

The following result is well known. (For example, see Petersen [23, Section 5.1].)

Lemma 6.10. Suppose that $Z$ is a standard Borel space with Borel probability measure $\mu$. If $\alpha=\left(A_{0}, A_{1}, \ldots, A_{n-1}\right)$ is any partition of $Z$ into Borel subsets, then $H(\alpha) \leq \log (n)$.

Finally we will make use of the following result, which is implicitly contained in Bowen [2]. Since Bowen does not state this result explicitly, we will briefly explain how to deduce Theorem 6.11 from the results in [2].

Theorem 6.11 (Bowen [2]). Let $2 \leq m \in \mathbb{N}$. Suppose that $G$ is a countable sofic group and that $Z \subseteq(m)^{G}$ is a $G$-invariant Borel subset with $\mu_{m}(Z)=1$. If I is a countable set and $\alpha=\left(A_{i} \mid i \in I\right)$ is a finite entropy $I$-generator of $Z$, then $H(\alpha) \geq \log (m)$.

Sketch Proof. Let $G$ be a countable sofic group and let $\Sigma$ be a sofic approximation of $G$. Suppose that $Z$ is a standard Borel $G$-space with $G$-invariant probability measure $\mu$. Then for each finite entropy generator $\alpha=\left(A_{i} \mid i \in I\right)$, Bowen defines a corresponding invariant $h(\Sigma, \alpha) \in \mathbb{R} \cup\{-\infty\}$ with the property that if $\beta$ is any other finite entropy generator, then $h(\Sigma, \beta)=h(\Sigma, \alpha)$. (For the fact that $h(\Sigma, \alpha)$ does not depend on the choice of $\alpha$, see Bowen [2, Theorem 2.1].) Furthermore, by Bowen [2, Proposition 5.3], we have that $h(\Sigma, \alpha) \leq H(\alpha)$. (To see this, let $\beta=\{X\}$ be the trivial partition in the statement of Proposition 5.3.)

Now consider the special case when $Z \subseteq(m)^{G}$ is a $G$-invariant Borel subset with $\mu_{m}(Z)=1$ and $\alpha=\left(A_{i} \mid i \in I\right)$ is a finite entropy $I$-generator of $Z$. Then, by Bowen [2, Theorem 8.1], we have that $h(\Sigma, \alpha)=\log (m)$ and so $H(\alpha) \geq \log (m)$. (The actual statement of Theorem 8.1 refers to $\left(m^{G}, \mu_{m}\right)$, but of course null sets can safely be ignored in this setting.) 
Proof of Theorem 6.2. If the result fails, then there exist integers $2 \leq n<m$ such that $F(G, m) \leq_{B} F(G, n)$. Hence, by Lemma 6.6, there exists a $G$-invariant Borel subset $Z \subseteq(m)^{G}$ with $\mu_{m}(Z)=1$ such that $Z$ admits an $n$-generator $\alpha=\left(A_{0}, A_{1}, \ldots, A_{n-1}\right)$. Applying Lemma 6.10 and Theorem 6.11, we have that

$$
\log (m) \leq H(\alpha) \leq \log (n),
$$

which is a contradiction.

\section{References}

[1] A. Andretta, R. Camerlo, and G. Hjorth, Conjugacy equivalence relation on subgroups. Fund. Math. 167 (2001), 189-212. Zbl 0978.03037 MR 1815088

[2] L. Bowen, Measure conjugacy invariants for actions of countable sofic groups. J. Amer. Math. Soc. 23 (2010), 217-245. Zbl 1201.37005 MR 2552252

[3] A. Connes, J. Feldman, and B. Weiss, An amenable equivalence relation is generated by a single transformation. Ergodic Theory Dynam. Systems 1 (1981), 431-450. Zbl 0491.28018 MR 662736

[4] R. Dougherty, S. Jackson, and A. S. Kechris, The structure of hyperfinite Borel equivalence relations. Trans. Amer. Math. Soc. 341 (1994), 193-225. Zbl 0803.28009 MR 1149121

[5] G. Elek and N. Monod, On the topological full group of a minimal Cantor $\mathbb{Z}^{2}$-system. Preprint 2012. arXiv:1201.0257v2 [math.DS]

[6] J. Feldman and C. C. Moore, Ergodic equivalence relations, cohomology, and von Neumann algebras. I. Trans. Amer. Math. Soc. 234 (1977), 289-324. Zbl 0369.22009 MR 0578656

[7] D. Gaboriau and R. Lyons, A measurable-group-theoretic solution to von Neumann's problem. Invent. Math. 177 (2009), 533-540. Zbl 1182.43002 MR 2534099

[8] R. Grigorchuk and K. Medynets, Topological full groups are locally embeddable into finite groups. Preprint 2012. arXiv:1105.0719v3 [math.GR]

[9] P. de la Harpe and A. Valette, La propriété (T) de Kazhdan pour les groupes localement compacts. Astérisque 158 (1989). Zbl 0759.22001 MR 1023471

[10] S. Jackson, A. S. Kechris, and A. Louveau, Countable Borel equivalence relations. J. Math. Log. 2 (2002), 1-80. Zbl 1008.03031 MR 1900547

[11] I. Kapovich and D. T. Wise, The equivalence of some residual properties of wordhyperbolic groups. J. Algebra 223 (2000), 562-583. Zbl 0951.20029 MR 1735163

[12] A. S. Kechris and B. D. Miller, Topics in orbit equivalence. Lecture Notes in Math. 1852, Springer-Verlag, Berlin 2004. Zbl 1058.37003 MR 2095154

[13] A. S. Kechris and Y. N. Moschovakis (Eds.), Cabal Seminar 76-77. Lecture Notes in Math. 689, Springer-Verlag, Berlin 1978. Zbl 0379.00001 MR 0526912

[14] A. Ju. Ol'šanskiǔ, Infinite groups with cyclic subgroups. Dokl. Akad. Nauk SSSR 245 (1979), 785-787; English transl. Soviet Math. Dokl. 20 (1979), 343-346. Zbl 0431.20025 MR 527709 
[15] A. Ju. Ol'šanskiŭ, An infinite group with subgroups of prime orders. Izv. Akad. Nauk SSSR Ser. Mat. 44 (1980), 309-321; English transl. Math. USSR-Izv. 16 (1981), 279-289. Zbl 0475.20025 MR 571100

[16] A. Yu. Ol'shanskii, On the problem of the existence of an invariant mean on a group. Uspekhi Mat. Nauk 35 (1980), no. 4, 199-200; English transl. Russian Math. Surveys 35 (1980), no. 4, 180-181. Zbl 0465.20030 MR 586204

[17] A. Yu. Ol'shanskii, Geometry of defining relations in groups. Math Appl. (Soviet Series) 70, Kluwer Academic Publishers, Dordrecht 1991. Zbl 0732.20019 MR 1191619

[18] A. Yu. Ol'shanskiǔ, Periodic factor groups of hyperbolic groups. Mat. Sb. 182 (1991), 543-567; English transl. Math. USSR-Sb. 72 (1992), 519-541. Zbl 0820.20044 MR 1119008

[19] A. Yu. Ol'shanskii,, On residualing homomorphisms and $G$-subgroups of hyperbolic groups. Internat. J. Algebra Comput. 3 (1993), 365-409. Zbl 0830.20053 MR 1250244

[20] A. Yu. Ol'shanskii and M. V. Sapir, Non-amenable finitely presented torsion-by-cyclic groups. Publ. Math. Inst. Hautes Études Sci. 96 (2002), 43-169 (2003). Zbl 1050.20019 MR 1985031

[21] N. Ozawa, There is no separable universal $\mathrm{II}_{1}$-factor. Proc. Amer. Math. Soc. 132 (2004), 487-490. Zbl 1041.46045 MR 2022373 (2004h:46074)

[22] V. G. Pestov, Hyperlinear and sofic groups: a brief guide. Bull. Symbolic Logic 14 (2008), 449-480. Zbl 1206.20048 MR 2460675

[23] K. Petersen, Ergodic theory. Cambridge Stud. Adv. Math. 2, Cambridge University Press, Cambridge 1983. Zbl 0507.28010 MR 833286

[24] S. Popa, Cocycle and orbit equivalence superrigidity for malleable actions of $w$-rigid groups. Invent. Math. 170 (2007), 243-295. Zbl 1131.46040 MR 2342637

[25] H. Rogers, Jr., Theory of recursive functions and effective computability. 2nd ed., MIT Press, Cambridge, MA, 1987. MR 886890

[26] D. Sonkin, CEP-subgroups of free Burnside groups of large odd exponents. Comm. Algebra 31 (2003), 4687-4695. Zbl 1046.20029 MR 1998023

[27] R. Steinberg, Some consequences of the elementary relations in $\mathrm{SL}_{n}$. In Finite groups coming of age (Montreal, Que., 1982), Contemp. Math. 45, Amer. Math. Soc., Providence, RI 1985, 335-350. Zbl 0579.20038 MR 822247

[28] S. Thomas, Popa superrigidity and countable Borel equivalence relations. Ann. Pure Appl. Logic 158 (2009), 175-189. Zbl 1162.03029 MR 2500091

[29] S. Thomas, Martin's conjecture and strong ergodicity. Arch. Math. Logic 48 (2009), 749-759. Zbl 1188.03032 MR 2563815

[30] S. Thomas, Continuous versus Borel reductions. Arch. Math. Logic 48 (2009), 761-770. Zbl 1184.03049 MR 2563816

Received January 19, 2010; revised January 4, 2011

S. Thomas, Mathematics Department, Rutgers University, 110 Frelinghuysen Road, Piscataway, New Jersey 08854-8019, USA

E-mail: sthomas@math.rutgers.edu 